\title{
Processing of Flange Measurements in Dimensional Control Tasks
}

\section{Introduction}

One of the roles of the surveying engineering is Dimensional Control. The term sets out a broad spectrum of issues related to industrial installation measurement and is used most often for petroleum industry.

Typical procedure performed here is determining the position of steel flange and its deformation. These issues are particularly important while working with objects undergoing construction, where prefabricate set needs to be fitted perfectly. A perfect example is an offshore oil rig, where due to the large number of high-pressure installations containing hazardous liquids, gases and chemical substances, works such as cutting and welding can be potentially dangerous. Joining the installation with the help of flanges and bolts does not involve such risk and simplify the renovation or the reconstruction process, without any losses related to the drilling halt.

Classic surveys are important part of the process due to high cost of preparation and transport of installations built on land, as well as significant consequences of the possible mismatch of prefabricated set. Determination of dimensions and existing deformations of the target platform's installation together with monitoring high compliance of prefabricate set with the project, which is being built, is a complex task. High, sub-millimeter precision while determining measured flange deformation is a key factor, decisive in joining the existing elements. Providing precise measurements translates to flange's sealing properties due to low level flexibility of a prefabricated sets.

There are no official records stating in what way such measurements and calculations should be performed. Terminology applied can differ depending on the location, internal standards of the company or habits of the person relying on the results. In the following publication terminology and methodology of the measurement process was based on the experience of surveyors performing works of this type for the purposes of companies from Great Britain. Since no relevant source of scientific data

\footnotetext{
* Rzeszów School of Engineering and Economics, Rzeszów, Poland
} 
was available, numerous conversations with professionals performing above mentioned measurements were taken into account. In fact, processing data in practice is often performed with the help of primitive graphical tools. Determining rough-in flange parameters is often made in CAD software or by, never published, algorithms created exclusively for selected companies. The goal of this paper is to define and describe a possible strategy for the flange measurement processing. This analysis resulted from actions focused on applying software solutions specifically designed for this type of tasks. Proposed methodology has been practically applied an tested in GEONET DC software

\section{Problems's Definition and Characteristic of Measurements}

Flange construction is shown in Figure 1. Detailed description of flange construction is available in [2]. Flange's dimensions are defined by various standards, for example [3]. For measurement purposes it is possible to make simplifications and assume that only two flange types exist: with flat and raise face. Three planes, essential while administering measurements, were marked with digits 1, 2 and 3 . In the case of a flange with a flat face (as shown in Figure 1b) face 1 is identical with face 2. For designers and flange manufacturers Tie Point (TP) is a point of particular interest. It is located at the intersection point of face 1 and flange main axis. Each flange is designed with concentrically located bolt holes. Adopted terminology distinguishes flanges with faces directed vertically up or down and calls them vertical, in contrast to horizontal ones.

a)

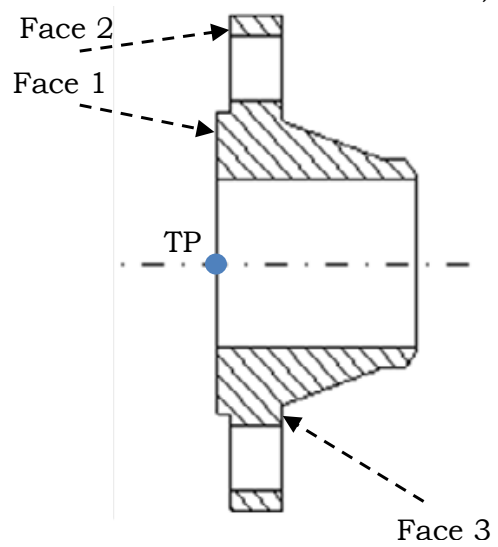

b)

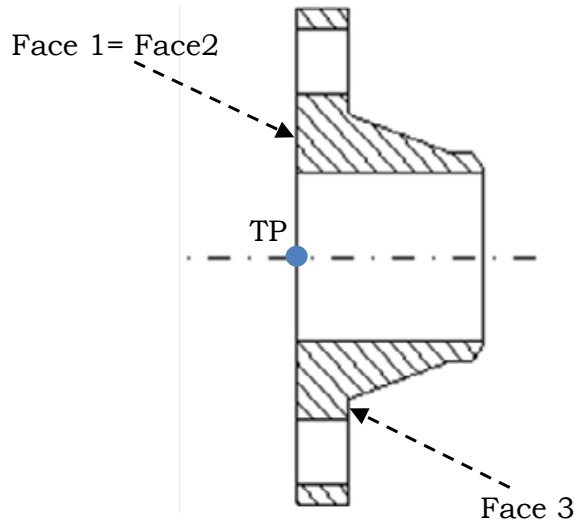

Fig. 1. Flange construction: a) raise face flange; b) flat face flange

Determining the direction in which face is directed to is significant. It is defined in different ways, depending on the flange position. 
For horizontal flanges (Fig. 2) direction of flange's front has been determined by two values:

$B_{H Z}$ - horizontal bearing-azimuth of normal face's vector,

$B_{V}$ - vertical bearing-angle between horizontal plane and normal vector.

For vertical flanges other values are determined:

$B_{N}$ - north bearing,

$B_{E}$ - east bearing.

While calculating directions and deflections, it is important to designate the sign of every angle. The following is shown in Figure 3, where graphical interpretation for numerous flange positions is exhibited.

Surveys are usually performed using classical total stations. Values of the measured angles and distances are processed and converted to local, 3D coordinate system. A measurements result is set of coordinates of measured points.

Task is about determining all parameters defining flange position together with its deformation, basing on point's coordinates in $x y z$ reference system:

- defining flange position and its Tie Point coordinates,

- defining face deflection to original or design direction $(\triangle B H Z$ and $\triangle B V$ or $\triangle B N$ and $\triangle B E$ ),

- defining bolts bearing and defining bolt central location,

- defining differences between existing and design dimensions; referring in particular to the face diameter $(R F 1, R F 2)$ and the bolt circle diameter $\left(R_{B}\right)$.

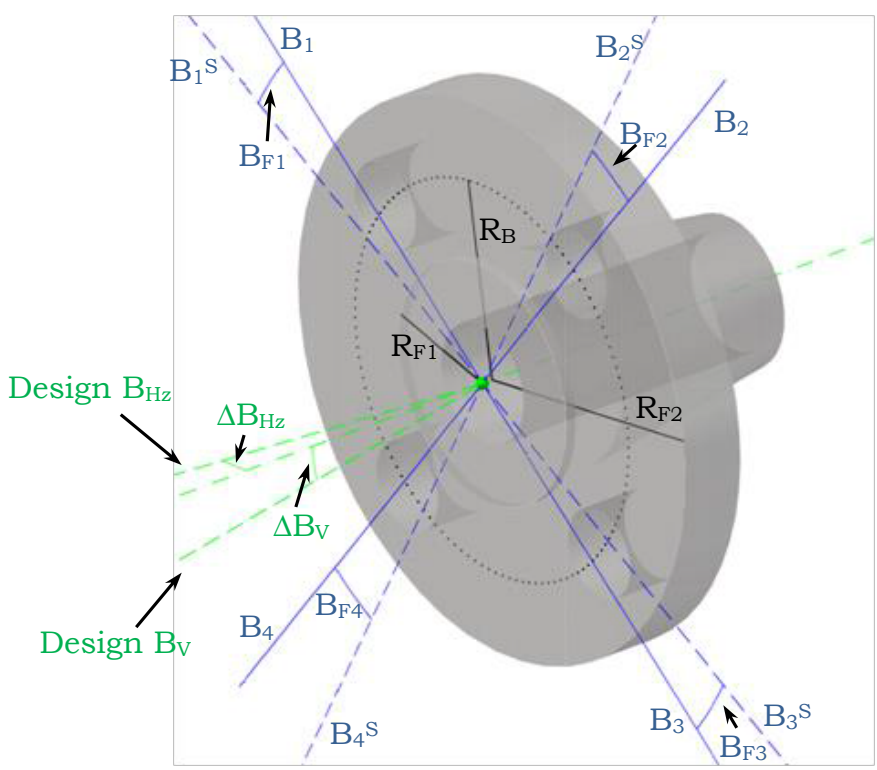

Fig. 2. Flanges parameters 


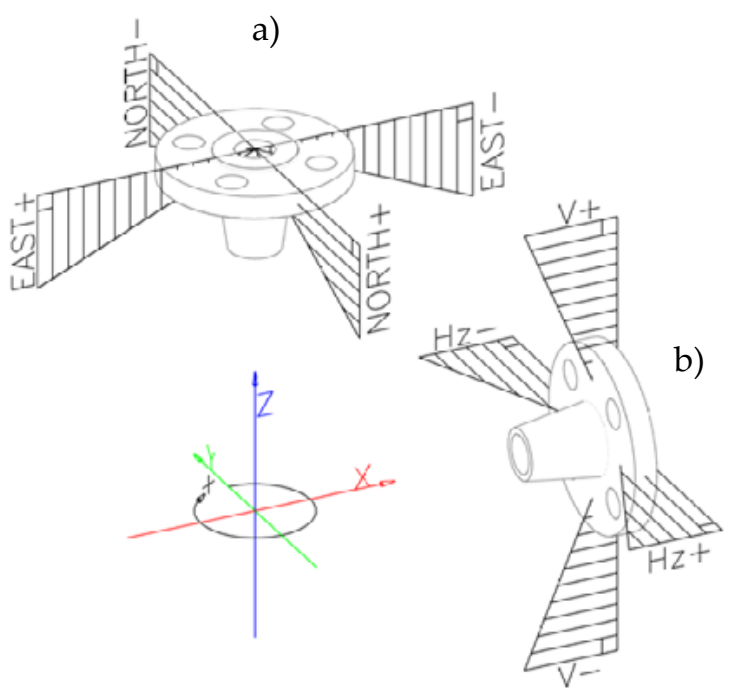

Fig. 3. Vertical and horizontal flange deflection: a) vertical flange; b) horizontal flange

\section{bolt's centers}

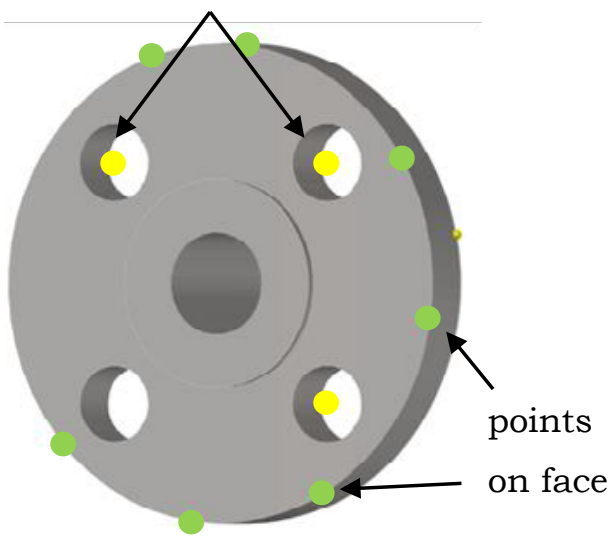

Fig. 4. Measured points

Depending on which flange part is available, measurement is performed with the use of points situated on the round edge of one of the faces 1, 2 or 3 (Fig. 4). Center's position of the visible bolts, flange thickness (between face 2 and 3), raise face thickness $T_{R F}$ (between face 1 and 2) is also computed. Edge's 3D coordinates and bolts diameter will be treated as direct measurement result in the following calculations. 


\section{Face fit - two-dimensional simplification}

First task is to perform the face fit into three-dimensional space using previously measured circle points. Such a circle is defined by the arrangement of two equations: sphere equation and the incisal plane equation which is going through a centre of a circle:

$$
\begin{gathered}
A\left(x-x_{S}\right)+B\left(y-y_{S}\right)+C\left(z-z_{S}\right)=0 \\
\left(x-x_{S}\right)^{2}+\left(y-y_{S}\right)^{2}+\left(z-z_{S}\right)^{2}=r^{2}
\end{gathered}
$$

where:

$$
\begin{array}{r}
x_{S^{\prime}} y_{S^{\prime}} z_{S} \text { - centre of a circle, } \\
A, B, C \text { - plane parameters, } \\
r \text { - radius of a circle. }
\end{array}
$$

Task can be solved be various methods [1,3]. Circle fit requires finding unknown $x_{S^{\prime}} y_{S^{\prime}} z_{S^{\prime}} A, B, C, r$ for the set of the minimum three, measured edges points, with determined $x, y, z$ coordinates. Standard approach suggests solving the above set of equations with the least squares method. After the linearization of the above task we can solve them determining the value of corrections to unknowns.

However such approach might not be the best. In practice, points not necessarily must lie precisely on the incisal plane - in fact, they are often drawn aside. Determining the precise flange edge might be troublesome (reason can be for example mechanical edge damage, poor accessibility as well as covering the flange with a thick layer of peeling paint). Applying standard least squares method, assuming the minimization of square observation amendments, does not guarantee that the centre of the circle will be precisely on the incisal plain. We cannot characterize the differences as considerable, however tests exhibited on empirical data shown that in practice it might be of millimeter value, which might be distorting to the results of calculations. A similar problem refers to the estimated value of the measured flange diameter. Also specifying the remaining parameters in three dimensional space might be complicated.

It is probably more appropriate to accommodate a different model, to determine Tie Point precisely in the face plane and at the same time define all components by manipulating data using rules of two dimensional local coordinate system of the described face.

Data computations are performed in stages. A primary consideration is to specify measured flange face parameters with the use of measured edge points. It is a standard task. Solving the equations as shown is proposed:

where:

$$
A\left(x-x_{P}\right)+B\left(y-y_{P}\right)+C\left(z-z_{P}\right)=0
$$

$x, y, z$-coordinates of measured points,

$x_{P^{\prime}} y_{P^{\prime}} z_{P}$ - coordinates of a any point on the surface,

$A, B, C$ - surface parameters. 
As a point $\left[x_{p}, y_{p^{\prime}} z_{p}\right]$ previously measured circle point centre of gravity can be assumed. The solution is to determine three unknowns $A, B, \mathrm{C}$. This can be executed by the standard least squares method. Designated plane normal vector $V=[A, B, C]$ at the same time defines the direction in which the flange face is positioned. To avoid numerical problems and to facilitate the further computation stages, the vector should be standardized. In addition, attention should be paid to the proper direction of $V$ vector. It should be directed "flange forward", from face 3 to face 1.

In the next stage all measuring points must be rotated in such a way as to set fitted plane horizontal, parallel to the $X Y$ plane. One of the possible methods of task accomplishing is making two rotations. The first involves rotating the entire system around the $Z$ axis, so as normal vector becomes parallel to $Z X$ plane. As the $\theta_{Z}$ angle of rotation, negative azimuth of $2 \mathrm{D}$ vector, created from components of $[A, B] V$ vector can be assumed.

Then:

$$
X_{Z}=R_{Z} \cdot X
$$

where $X$ represents a rotation point expressed in the form of matrix and $R_{z}$ is a matrix of rotation. Figuring out the formula for matrix of rotation $R_{Z^{\prime}}$ then:

$$
\left[\begin{array}{l}
X_{Z} \\
Y_{Z} \\
Z_{Z}
\end{array}\right]=\left[\begin{array}{ccc}
\cos \left(\theta_{Z}\right) & \sin \left(\theta_{Z}\right) & 0 \\
-\sin \left(\theta_{Z}\right) & \cos \left(\theta_{Z}\right) & 0 \\
0 & 0 & 1
\end{array}\right]\left[\begin{array}{l}
X \\
Y \\
Z
\end{array}\right]
$$

where $\theta_{Z}$ represents the angle of rotation to $Z$ axis, $X_{Z^{\prime}} Y_{Z^{\prime}} Z_{Z}$ are rotated coordinates here.

The second rotation, this time around $Y$ axis should be performed in such a way, to make face parallel to $X Y$ plane. $\theta_{Y}$ could have been assumed as:

$$
\theta_{Y}=p-\arccos (C)
$$

For a such defined angle, rotation formula will be then:

$$
X_{R}=R_{Y} \cdot X_{Z}
$$

where $X_{R}$ denotes the coordinates of a point after the turning and $R_{Y}$ will be expressing matrix of rotation around the $Y$ axis.

The above equation can be described as:

$$
\left[\begin{array}{c}
X_{R} \\
Y_{R} \\
Z_{R}
\end{array}\right]=\left[\begin{array}{ccc}
\cos \left(\theta_{Y}\right) & 0 & -\sin \left(\theta_{Y}\right) \\
0 & 1 & 0 \\
\sin \left(\theta_{Y}\right) & 0 & \cos \left(\theta_{Y}\right)
\end{array}\right]\left[\begin{array}{c}
X_{Z} \\
Y_{Z} \\
Z_{Z}
\end{array}\right]
$$


The joined rotations are then:

$$
X_{R}=R_{Y} \cdot R_{Z} \cdot X
$$

All points are rotated: on the edge of the plane (on the circle), and bolt centers. Since 3D circle's incisal plane due to rotation becomes parallel to the XY plane further computations can be performed, referring to two dimensional space. Projection of the measured points on to the $X, Y$ plane means simply rejection of the third, $\mathrm{Z}$ coordinate.

\section{Circle Fit}

In the rotated $X, Y$ points (which determine the round edge of the measured plane) two dimensional circle is fitted. Standard circle fit algorithm of the least square method can be here applied. Thanks to standard two dimensional circle space formula, equations will be then:

$$
\left(X-X_{C}\right)^{2}+\left(Y-Y_{C}\right)^{2}=r^{2}
$$

After the linearization, the above set of equations can be solved with the least squares method. The result will be the value of the radius and the flat circle of the centre coordinates $C_{2 \mathrm{D}}=\left[X_{C^{\prime}}, Y_{C}\right]$. To obtain coordinates of the circle centre in three dimensional coordinate system, point $C_{2 \mathrm{D}}^{\prime}=\left[X_{C^{\prime}}, Y_{C^{\prime}} 0\right]$ needs to be converted back to three dimensional system. Appropriate models (equations 4 and 7) in the reverse order must be applied: first rotation around $\mathrm{Y}$ axis, and then around the $\mathrm{Z}$ axis together with the opposite value of the angle $\theta_{Y}$ and $\theta_{Z}$ :

$$
C_{3 \mathrm{D}}=R_{Z} R_{Y} C_{2 \mathrm{D}}^{\prime}
$$

where rotation matrices $R_{Z}$ and $R_{Y}$ based on the angles: $\theta_{Y}^{\prime}=-\theta_{Y}, \theta_{Z}^{\prime}=-\theta_{Z}$ and $C_{3 \mathrm{D}}$ point expresses three dimensional coordinates of the centre of the fitted circle located precisely in the incistial plane.

\section{TP Coordinates Computation}

Tie Point coordinates computation requires transferring $C_{3 \mathrm{D}}$ centre of the fitted circle along normal vector to plane. Transferring length is equal to flange thickness, named $T_{R F}$ or $T+T_{R F}$ depending on to which one is being measured. TP coordinates will be then:

$$
\left[\begin{array}{c}
X_{T P} \\
Y_{T P} \\
Z_{T P}
\end{array}\right]=\left[\begin{array}{l}
X_{3 \mathrm{D}} \\
Y_{3 \mathrm{D}} \\
Z_{3 \mathrm{D}}
\end{array}\right]+d\left[\begin{array}{c}
A \\
B \\
C
\end{array}\right] \begin{gathered}
d=0 \text { if face } 1 \text { is measured } \\
d=T_{R F} \text { if face } 2 \text { is measured } \\
d=T+T_{R F} \text { if face } 3 \text { is measured }
\end{gathered}
$$

where:

$X_{T P^{\prime}}, Y_{T P^{\prime}} Z_{T P}-$ TP coordinates,

$A, B, C$ - standardized normal vector directed "flange forward". 


\section{Deflections from Design Bearing}

Direction of the flange front (flange bearing) is described by specifying appropriate values, based on the direction of the vector normal to the plane. For horizontal flange, assumed length of $[A, B, C]$ vector equals 1 :

$$
B H Z=\text { azimuth }[A, B], \quad B V=\arcsin (C)
$$

For vertical flange:

$$
B_{N}=-\arctan (B / C), \quad B_{E}=-\arctan (A / C)
$$

Deflections $\left(\Delta B_{H Z}\right.$ and $\Delta B_{V}$ or $\Delta B_{N}$ and $\left.\Delta B_{E}\right)$ is calculated as difference between design bearing and $B_{H Z}$ and $B_{V}$ or $B_{N}$ and $B_{E}$ depending on the flange direction (horizontal or vertical).

\section{Bolt Circle Radius and Bolt Bearing}

The next step is to calculate the radius of the circle described by the bolts centers. Usually, the protruding ends are measured and measured points are not situated directly on the plane. In practice, length of the protruding ends might be different, so the measurement is not always parallel to the face. That is why direct 3D circle fit would not be correct.

It is advised to project rotated bolts on the designated face (solution previously discussed) and re-examine issues in two-dimensional space. Because the bolts have already been transformed and leveled, calculation of the radius and the centre of the circle can be performed analogous to a round edge fit. The result of such calculation will be the point, of the centre of a circle described as $C_{B 2 \mathrm{D}}=\left[X_{C B^{\prime}} Y_{C B}\right]$ with $R_{B}$ radius. For control purposes difference between fitted circles centers $C_{B 2 D}$ and $C_{2 D}$ should be checked:

$$
d_{X}=X_{C B}-X_{C} d_{Y}=Y_{C B}-Y_{C}
$$

When proper measured, $d_{X}$ and $d_{Y}$ value should be quite small.

Relevant component describing bolt holes position is bolt bearing, which is illustrated in Figure 2. Summarical $B_{F}$ bearing is described as mean difference $\left(B_{F 1-4}\right)$ between the measurement from $C_{B 2 D}$ centre to $B_{k}(k$-bolt center), and typical direction for every bolt $\left(B_{k}^{s}\right)$.

Directions are calculated starting from zero direction $\left(B_{0}\right)$, depending on the orientation:

- for horizontal flange, it will be horizontal plane intersection, going through the centre of a circle to the flange face,

- for vertical flange it will be vertical plane intersection (with N-S direction) going through the centre of measured flange circle. 
When installing, the flange is set so that the zero direction is situated precisely between two holes. Assuming that the number of holes is always divisible by 4 (due to flange construction), the default direction value would be defined as:

$$
B_{k}^{S}=\frac{\pi}{n}+\frac{(k-1) \cdot 2 \pi}{n}=\frac{\pi(2 k-1)}{n}
$$

where:

$n$ - the number of all holes,

$k$ - number of a given hole, starting from the zero direction.

The value of the real $B_{k}$ will be identified on the basis of measured and rotated by formulas 4 and 7 bolt holes centers. It exhibits the difference between $B_{0}$ and $B_{k}$ azimuth. Final $B_{F}$ is exhibited here:

$$
B_{F}=\frac{\sum_{k=1}^{m} B_{k}^{S}-B_{k}}{m}
$$

where $m$ - number of all bolts centers measured.

\section{Conclusions}

Precise flange's position and deflection measurements has proven to be invaluable in reducing preparation and transport costs of installations built for oil platforms. Proposed algorithm of processing such measurements allows for all flange parameters precise determination. Computations then can be performed easily, referring to two dimensional space. Method is already implemented and tested in GEONET DC software which is use in practice to process such measurements in Oil and Gas industry.

\section{References}

[1] Ahn S.J., Rauh W., Warnecke H.J.: Least-squares orthogonal distances fitting of circle, sphere, ellipse, hyperbola, and parabola. Pattern Recognition, vol. 34, issue 12, December 2001, pp. 2283-2303.

[2] Nayyar M.: Piping Handbook. $7^{\text {th }}$ ed. McGraw-Hill, New York 1999.

[3] Pipe Flanges and Flanged Fittings: NPS 1/2 through NPS 24 Metric/Inch Standard. The American Society of Mechanical Engineering, New York 2013.

[4] Shakarji C.: Least-Squares Fitting Algorithms of the NIST Algorithm Testing System. Journal of Research of the National Institute of Standards and Technology, vol. 103, no. 6, November-December 1998, pp. 633-641. 